\title{
Phenyl-Linked Anthracene-Based Macrocycles with Geometrically Tunable Optical Properties
}

\author{
Ming-Guang Rong ${ }^{a}$ (D) \\ Junting Wang ${ }^{a}$ (i) \\ Kam-Hung Low ${ }^{a}$ (i) \\ Junzhi Liu*a (iD)

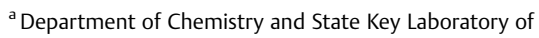 \\ Synthetic Chemistry, The University of Hong Kong, Pokfulam \\ Road, Hong Kong, P. R. China \\ juliu@hku.hk
}

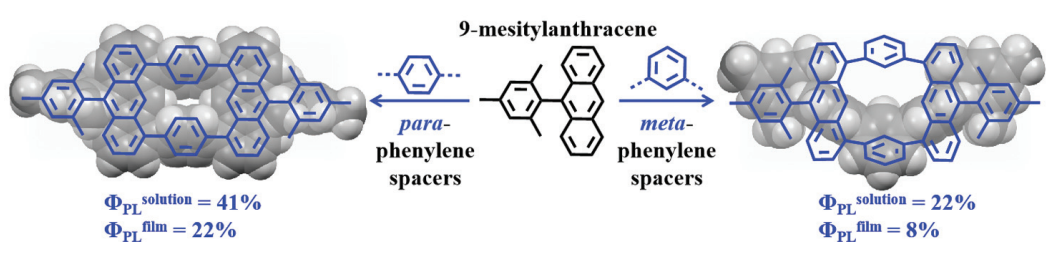

Received: 30.10 .2020

Accepted after revision: 11.11.2020

DOI: 10.1055/s-0040-1721729; Art ID: om-20-0037sc

License terms: (c)

(c) 2020. The Author(s). This is an open access article published by Thieme under the terms of the Creative Commons Attribution-NonDerivative-NonCommercial License, permitting copying and reproduction so long as the original work is given appropriate credit. Contents may not be used for commercial purposes, or adapted, remixed, transformed or built upon. (https://creativecommons.org/licenses/by-nc-nd/4.0/)

Abstract Anthracene has been widely explored because of its intrinsic photophysical and photochemical properties. Here, two novel anthracene-based macrocycles ( $\mathbf{1}$ and $\mathbf{2}$ ) were designed and synthesized with para- and meta-phenylene spacers. X-ray crystallographic analysis demonstrates that compound $\mathbf{1}$ with para-phenylene spacers adopts a nearly planar structure, while compound 2 with meta-phenylene spacers displays a V-shaped geometry. The photophysical properties of the resultant macrocycles, which are structural isomers, are well studied using photoluminescence spectra and time-resolved absorption spectra, which are further corroborated by density functional theory calculations. The optical properties of these two novel macrocycles can be finely tuned via their geometries.

Key words anthracene, macrocycles, geometrically, optical properties, fluorescence

\section{Introduction}

Well-defined $\pi$-conjugated macrocycles have attracted considerable attention from researchers because they not only are theoretically and experimentally interesting, but they also demonstrate potential applications in carbon-based nanoelectronics. ${ }^{1-8}$ In particular, the cyclo-p-phenylenes have been well developed during the past decade, due to their unique structures, optoelectronic properties, and supramolecular host-guest behavior. ${ }^{9-15}$ Anthracene is a versatile synthon that is widely used to synthesize attractive and functional molecules in organic electronics, due to its molecular panel-like shape and excellent photophysical properties. ${ }^{16-22}$ Moreover, the feasibility of chemical modification of the rigid anthracene backbone makes anthracene and its derivatives very useful building blocks for the synthesis of fascinating cyclic structures. ${ }^{23-38}$ In 2007, Toyota and coworkers reported the synthesis of the cyclic 1,8-anthryleneethynylene dimer with two acetylene linkers (Figure 1) through the Sonogashira coupling. ${ }^{39-41}$ In 2016, the same group demonstrated the synthesis of a novel $\pi$-conjugated macrocyclic compound consisting of six 2,7-anthrylene units by Ni-mediated Yamamoto coupling (Figure 1). ${ }^{26,27}$ A macrocyclic diphenylanthracene dimer was successfully synthesized by lyoda et al. using the electron-transfer oxidation of Lipshutz cuprate in 2018 (Figure 1). ${ }^{42}$ Although these achievements made progress, the synthesis of smaller macrocycles with anthracene units still remains a challenge, such as a phenyl-linked 1,8-anthracene dimer, due to the high strain at the 1,8 -position and the rigid $\pi$-frame of anthracene.

Herein, we demonstrated the synthesis of two novel anthracene-based macrocycles with para- and meta-phenylene linkers: the structural isomers $\mathbf{1}$ and $\mathbf{2}$ (Scheme 1). The chemical structures of $\mathbf{1}$ and $\mathbf{2}$ are unambiguously confirmed by X-ray crystallographic analysis. The anthracene units in macrocycle $\mathbf{1}$ adopt a nearly planar structure, while the

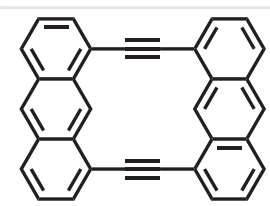

Toyota, 2007 and 2019<smiles></smiles>

lyoda, 2018

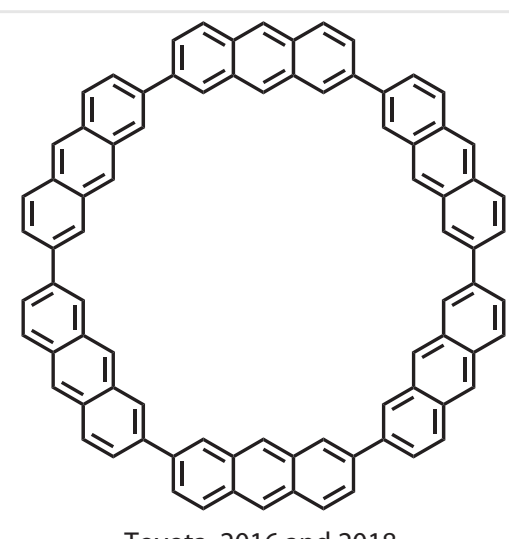

Toyota, 2016 and 2018

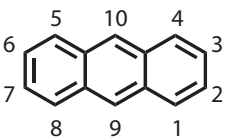

Figure 1 Examples of anthracene-based macrocycles. 


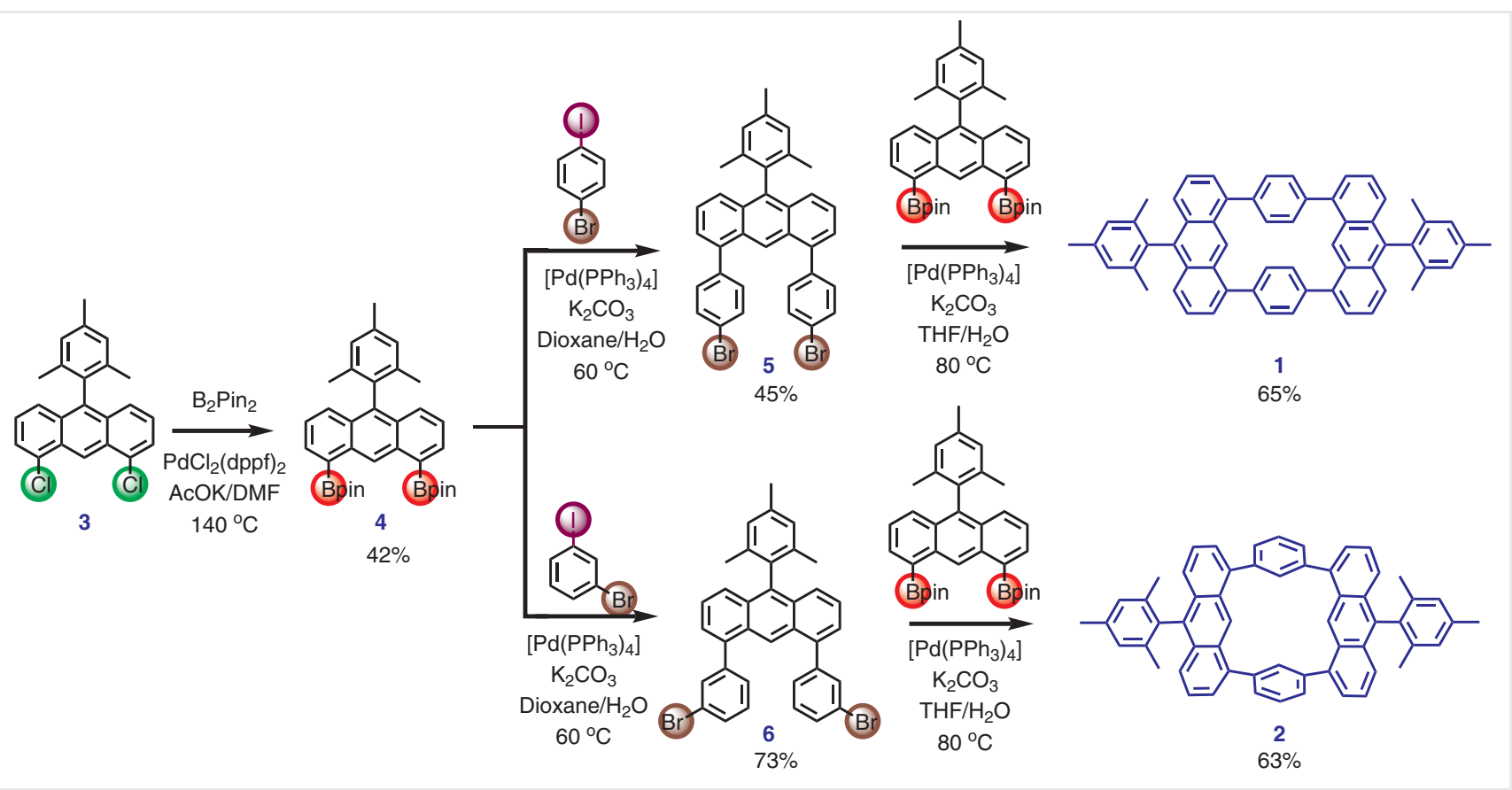

Scheme 1 Synthetic routes toward anthracene-based macrocycles $\mathbf{1}$ and $\mathbf{2}$.

anthracene units in $\mathbf{2}$ possess a slightly curved geometry due to its highly twisted geometry (Figure 2). Interestingly, the structural isomers $\mathbf{1}$ and $\mathbf{2}$ demonstrate totally different optical and electronic properties. The maximum absorption and fluorescence peaks of $\mathbf{2}$ are blue-shifted compared to those of compound $\mathbf{1}$ due to the highly strained structure, which leads to the decreased conjugation in $\mathbf{2}$. Accordingly, the photoluminescence quantum yield (QY; $\Phi_{\mathrm{PL}}$ ) of $\mathbf{2}(22 \%)$ is lower than that of $\mathbf{1}$ (41\%) both in solution and solid films (in $40 \mathrm{wt} \%$ PMMA, 1: $\Phi_{\mathrm{PL}}{ }^{\text {film }}=22 \% ; 2: \Phi_{\mathrm{PL}}{ }^{\text {film }}=8 \%$ ). However, the fluorescence lifetimes of $\mathbf{1}(\tau=6.15 \mathrm{~ns})$ and $\mathbf{2}$ ( $\tau=6.19 \mathrm{~ns}$ ) are comparable with each other. Thereby, our work reported herein is particularly attractive because it not only allows the synthesis of highly strained macrocycles with 1,8-anthracene units, but also provides the possibility to finetune their optoelectronic properties by controlling the geometry.

\section{Results and Discussion}

The synthetic routes toward anthracene-based macrocycles $\mathbf{1}$ and $\mathbf{2}$ are depicted in Scheme 1. The key building block 1,8-diborate-10-mesitylanthracene (4) was synthesized from 1,8-dichloro-10-mesitylanthracene $(3)^{43}$ through the palladium-catalyzed Miyaura borylation reaction with bis (pinacolato)diboron in $42 \%$ yield. Subsequently, the selective Suzuki coupling of compound 4 with 1 -bromo-4-iodobenzene or 1-bromo-3-iodobenzene afforded compounds $\mathbf{5}$ and $\mathbf{6}$ in the yield of $45 \%$ and $73 \%$, respectively. Finally, the anthracenebased macrocycles $\mathbf{1}$ and $\mathbf{2}$ were successfully obtained through the Suzuki coupling of $\mathbf{5}$ and $\mathbf{6}$ with compound $\mathbf{4}$ in excellent yields of $65 \%$ and $63 \%$, respectively. The chemical structures of $\mathbf{1}$ and $\mathbf{2}$ were fully characterized by ${ }^{1} \mathrm{H}$ and ${ }^{13} \mathrm{C}$ NMR measurements (Figures S3-S14) and the high-resolution mass spectroscopy (Figures S15-S21). ${ }^{44,45}$

The crystals of macrocycles $\mathbf{1}$ and $\mathbf{2}$ were grown by slow evaporation of their solution in methanol/toluene and hexane/dichloromethane, respectively, allowing characterization of their structural features by X-ray single crystal analysis. The anthracene groups in macrocycle $\mathbf{1}$ possess a nearly planar geometry, in which the phenyl rings twist out of the anthracene plane with a dihedral angle of $58.62^{\circ}$ (Figure 2a, b). In the solid state of macrocycle $\mathbf{2}$, the anthracene units and phenyl rings twist each other with the dihedral angles of $72.55^{\circ}$ to $79.89^{\circ}$ (Figure 2c, d). Interestingly, due to the high strain in macrocycle 2 , the anthracene units also slightly twist out of the plane with a dihedral angle of $4.48^{\circ}$ (Figure $2 \mathrm{~d}$ ), which is similar to those of the 1,8-disubstituted anthracene compounds in the literature. ${ }^{46}$ Due to the presence of the sterically hindered mesityl groups and the twisted para- (1) and meta- (2) phenylene spacers, no intermolecular $\pi-\pi$ stacking interaction can be formed between the adjacent anthracene moieties (Figures S1 and S2).

The electrochemical behaviors of macrocycles $\mathbf{1}$ and $\mathbf{2}$ in anhydrous dichloromethane $\left(\mathrm{CH}_{2} \mathrm{Cl}_{2}\right)$ were investigated by means of cyclic voltammetry; the results are depicted 
a)

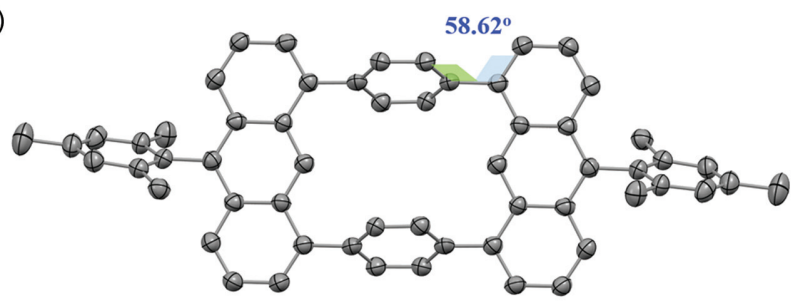

b)

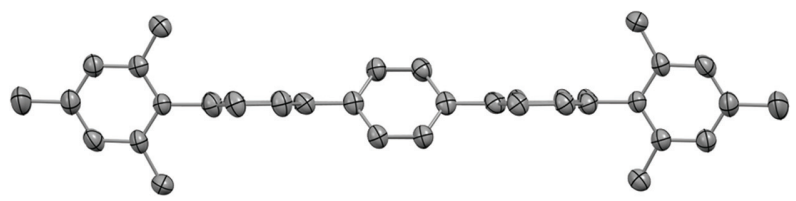

c)

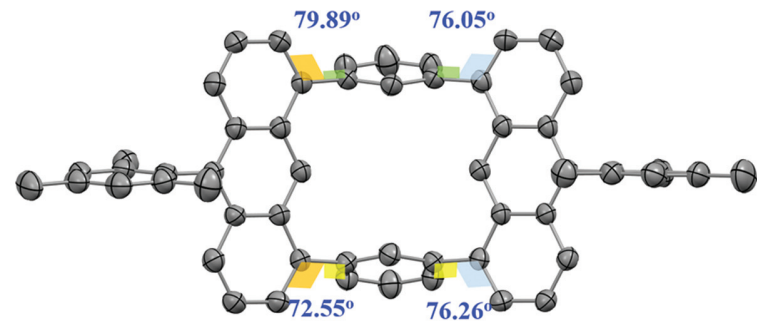

d)

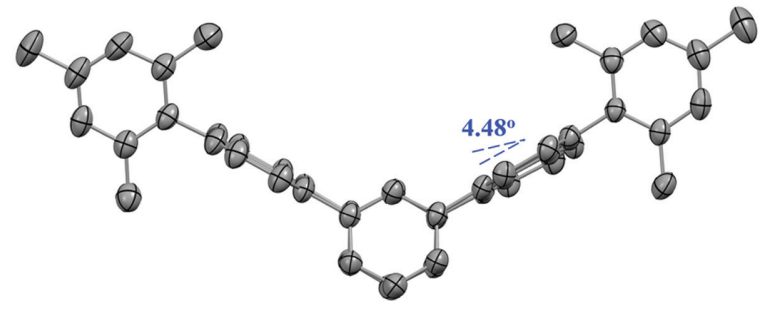

Figure 2 X-ray crystal structures of macrocycles $\mathbf{1}$ and $\mathbf{2}$. All the hydrogen atoms and solvent molecules are omitted. (a, b) Top and side views of $\mathbf{1}$. (c, d) Top and side views of $\mathbf{2}$.
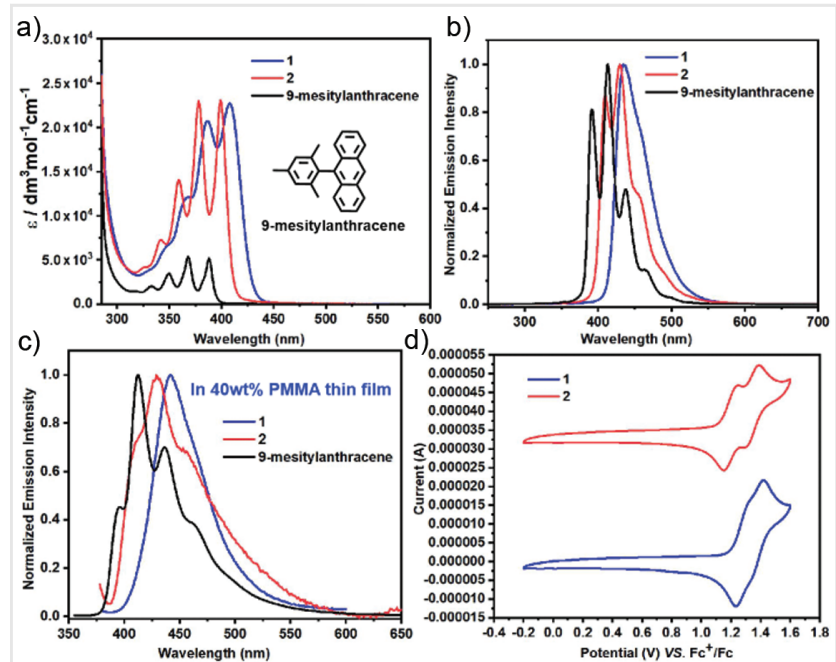

Figure 3 UV-vis absorption (a) and fluorescence (b) spectra of 9 mesitylanthracene, $\mathbf{1}$ and $\mathbf{2}$ in toluene (concentration: $5 \times 10^{-5} \mathrm{M}$ ). (c) Fluorescence spectra of 9-mesitylanthracene, 1 and 2 in 40 wt\% PMMA thin film. (d) Cyclic voltammetry of $\mathbf{1}$ and $\mathbf{2}$ measured in $\mathrm{CH}_{2} \mathrm{Cl}_{2}(0.1 \mathrm{M}$ $\mathrm{n}-\mathrm{Bu}_{4} \mathrm{NPF}_{6}$ ) at the scan rate of $50 \mathrm{mV} / \mathrm{s}$.

in Figure 3d. Macrocycle 1 showed two reversible oxidation processes at the onset potential of 1.20 and $1.23 \mathrm{~V} \mathrm{vs}$. $\mathrm{Fc}^{+} / \mathrm{Fc}$ (Figure 3d), respectively. For macrocycle $\mathbf{2}$, which exhibited a similar pattern to compound 1, two reversible oxidation processes were observed with the onset potential of 1.12 and $1.21 \mathrm{~V}$ vs. $\mathrm{Fc}^{+} / \mathrm{Fc}$ (Figure 3d), respectively. Accordingly, the HOMO energy levels are estimated to be -5.45 and

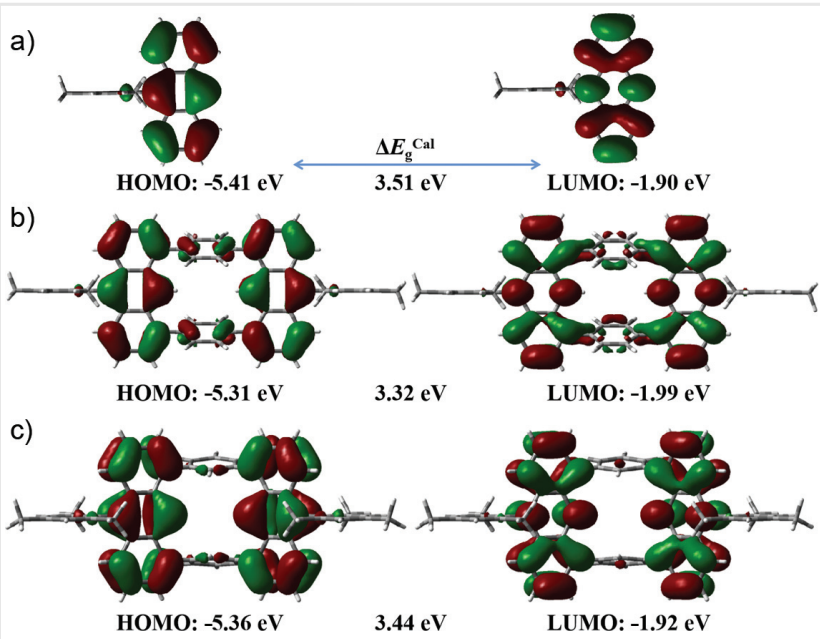

Figure 4 Calculated molecular orbitals and energy diagrams of 9mesitylanthracene (a), 1 (b), and $\mathbf{2}$ (c).

$-5.53 \mathrm{eV}$ for $\mathbf{1}$ and $\mathbf{2}$, respectively. In addition, the experimental results are well supported by the density functional theory calculations (Figure 4). In comparison with 9mesitylanthracene (Figure 4a), the energy gaps of macrocycles $\mathbf{1}$ and $\mathbf{2}$ are smaller than that of monomer 9 -mesitylanthracene due to the $\pi$-expanded conjugation. The HOMO and LUMO in macrocycle $\mathbf{1}$ with para-phenylene spacers are mainly localized on the anthracene units and partially on the para-phenylene linkers (Figure $4 \mathrm{~b}$ ). In contrast, the HOMO and LUMO of macrocycle 2 with metaphenylene spacers are fully localized over the anthracene 
groups, owing to the interruption of conjugation by the meta-phenylene spacers, as well as the deplanarization (Figure 4c). Accordingly, the energy gap of macrocycle 2 $\left(\Delta E_{\mathrm{g}}{ }^{\mathrm{Cal}}=3.44 \mathrm{eV}\right)$ is larger than that of its structural isomer $\mathbf{1}$ $\left(\Delta E_{\mathrm{g}}{ }^{\mathrm{Cal}}=3.32 \mathrm{eV}\right)$, with a lower HOMO and a higher LUMO (Figure 4). The photophysical properties of macrocycles 1 and $\mathbf{2}$ were investigated by UV-vis absorption and fluorescence spectroscopy in aerated toluene solution $\left(5 \times 10^{-5}\right.$ M; Figure 3). Compounds 9-mesitylanthracene, 1, and 2 display similarly shaped UV-vis absorption patterns, in which four absorption peaks ranging from 325 to $450 \mathrm{~nm}$ can be observed. Compared to the monomer 9-mesitylanthracene, the maximum absorption peaks for the anthracene-based macrocycles $\mathbf{1}$ and $\mathbf{2}$ were red-shifted with high molar extinction coefficients $\left(\varepsilon_{\max }=2.28-2.32 \times 10^{4} \mathrm{M}^{-1} \mathrm{~cm}^{-1}\right)$, owing to their narrower HOMO/LUMO gaps (Figure 3a). Interestingly, we found that the photophysical properties could be tuned by different geometries of the macrocycles; this can be illustrated by the absorption and emission maxima of structural isomers $\mathbf{1}$ and $\mathbf{2}$. For instance, the absorption maximum peak of macrocycle $\mathbf{2}$ (398 $\mathrm{nm}$ ) with meta-phenylene spacers exhibits a blue-shift of $10 \mathrm{~nm}$ compared to that of its respective isomer 1 (408 nm) with para-phenylene spacers (Figure $3 \mathrm{a}$ ), which is attributed to its enlarged energy gap. Macrocycle 2 displays a maximum emission peak at 428 and $430 \mathrm{~nm}$ in the solution and solid state (Figure 3b, c), respectively, with a blue-shift of 6 and $11 \mathrm{~nm}$ compared with 1 . Two shoulder emission peaks at 408 and $457 \mathrm{~nm}$ were also observed for $\mathbf{2}$; while macrocycle $\mathbf{1}$ exhibits a broad emission peak which centered at $434 \mathrm{~nm}$ due to its rigid structure. Notably, a smaller Stokes shift of macrocycle $\mathbf{1}(26 \mathrm{~nm})$ in toluene can be observed compared with that of $\mathbf{2}(30 \mathrm{~nm})$. Furthermore, the solution photoluminescence $\mathrm{QY}$ of macrocycle $1\left(\Phi_{\mathrm{QY}}=41 \%\right)$ with paraphenylene spacers is much higher than that of its structural isomer $2\left(\Phi_{\mathrm{QY}}=22 \%\right)$ with meta-phenylene spacers and the solid-state QY of 1 (in 40 wt\% PMMA thin film, $\Phi_{\mathrm{OY}}{ }^{\text {film }}=22 \%$ ) is also superior to that of $\mathbf{2}$ ( $\Phi_{\mathrm{QY}}{ }^{\text {film }}=8 \%$ ), indicating less structural relaxation of $\mathbf{1}$ both in solution and solid state. The fluorescence lifetimes of 9-mesitylanthracene, $\mathbf{1}$, and $\mathbf{2}$ are similar, reaching $\tau=7.02$, 6.15 , and 6.19 ns, respectively.

The excited-state nature of macrocycles $\mathbf{1}$ and $\mathbf{2}$ was examined by nanosecond time-resolved absorption (ns-TA) spectroscopy (Figure 5). The experiments were conducted in aerated toluene via $355 \mathrm{~nm}$ excitation of 9-mesitylanthracene, 1, and 2. Compared to monomer 9-mesitylanthracene (Figure 5a), the macrocycles $\mathbf{1}$ and $\mathbf{2}$ show totally different ns-TA absorption profiles (Figure 5), revealing that they are not derived from the same excited state. For instance, 9-mesitylanthracene shows two positive absorption bands at 407 and $429 \mathrm{~nm}$, while macrocycles 1 and 2 feature intense broad absorption bands at ca. $410-580 \mathrm{~nm}$. Compared to 9-mesitylanthracene, the two macrocyclic compounds exhibit more intense negative absorption bands originating from ground-state bleaching in the spectral range of $360-420 \mathrm{~nm}$. On the other hand, macrocycles $\mathbf{1}$ and $\mathbf{2}$ also display differences in their ns-TA spectra (Figure $5 \mathrm{~b}, \mathrm{c}$ ). The negative absorption of compound $\mathbf{2}$ is well resolved, but not for the broad and featureless profiles found in its structural isomer 1. The difference shown in their ns-TA spectra suggests different electronic configurations of the excited states between these two compounds, and is also consistent with their different emission properties in which compound $\mathbf{1}$ displays broad and sharp emission bands but $\mathbf{2}$ shows vibronic-structured emission profiles (Figure $3 \mathrm{~b}$ ). Kinetic studies at selected wavelengths of these ns-TA spectra were also carried out with decay time constants of 329 (9-mesitylanthracene), 232 (1), and 274 (2) ns.

\section{Conclusions}

In conclusion, we report the synthesis of two novel macrocycles $\mathbf{1}$ and $\mathbf{2}$, in which the 1,8-anthracene units are linked with the para- and meta-phenylene units, resulting in the formation of their structural isomers. From the X-ray
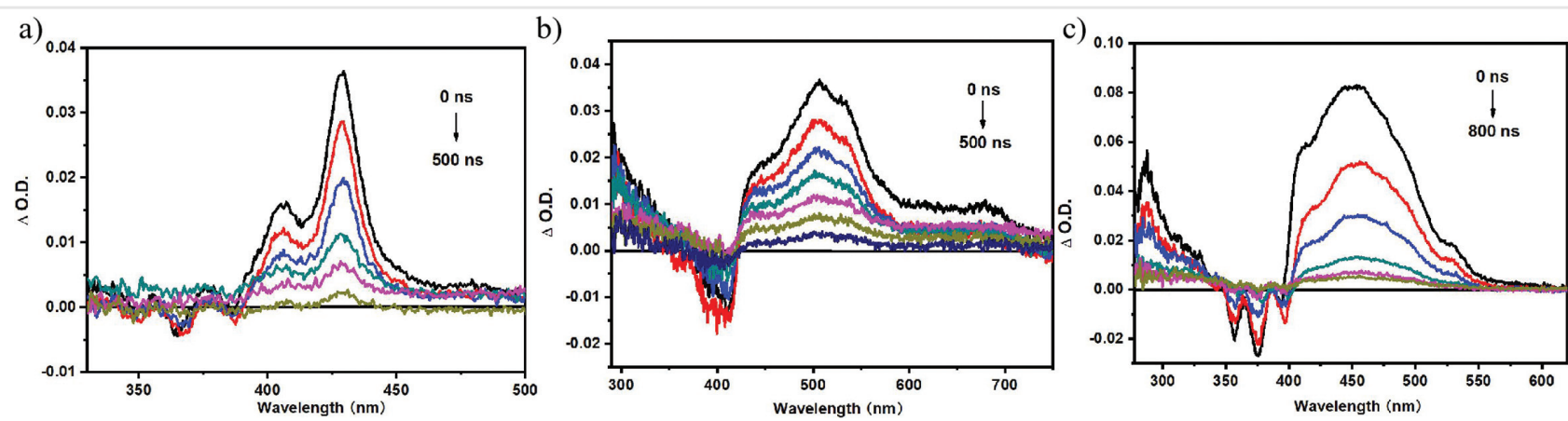

Figure 5 Nanosecond time-resolved absorption (ns-TA) spectra of 9-mesitylanthracene (a), macrocycles $\mathbf{1}$ (b), and $\mathbf{2}$ (c) in toluene (concentration: $\left.5 \times 10^{-5} \mathrm{M}\right)$ at $355 \mathrm{~nm}$ laser pulse excitation. 
crystallographic analysis, the anthracene units in macrocycle 1 possess nearly planar structures; however, the anthracene units in $\mathbf{2}$ show a slightly curved geometry with a dihedral angle of $4.48^{\circ}$. Notably, the structural isomers 1 and 2 display striking difference in their photophysical properties, in which the macrocycle $\mathbf{1}$ with para-phenylene spacers demonstrates a much higher $\Phi_{\mathrm{PL}}$ value both in solution (41\%) and solid state (22\%) compared with those of $\mathrm{V}$-shaped macrocycle $\mathbf{2}$ with meta-phenylene spacers (for $\mathbf{2}$ : $\Phi_{\mathrm{PL}}{ }^{\text {solution }}=22 \%$; $\Phi_{\mathrm{PL}}{ }^{\text {film }}=8 \%$ ). Our work reported herein offers the possibility to fine-tune photophysical properties by controlling the geometry of macrocycles.

\section{Funding Information}

This work was supported by Research Grants Council, University Grants Committee, Hong Kong (HKU 27301720). $\mathrm{J}$. Liu is grateful for the funding support from Innovation and Technology Commission, Hong Kong to the SKL.

\section{Acknowledgment}

We thank Dr. Dongling Zhou (The University of Hong Kong, HKU) for the time-resolved absorption (ns-TA) measurements. We are grateful for the UGC funding administered by HKU for supporting the Time-of-Flight Mass Spectrometry Facilities under the Support for Interdisciplinary Research in Chemical Science and HKU University Development Fund for funding the X-Ray Diffractometer Facilities. We acknowledge the computer cluster (HPC2015) of HKU for generous allocations of computing resources.

\section{Supporting Information}

Supporting Information for this article is available online at https://doi.org/10.1055/s-0040-1721729.

\section{References And Notes}

(1) Tahara, K.; Tobe, Y. Chem. Rev. 2006, 106, 5274.

(2) Iyoda, M.; Yamakawa, J.; Rahman, M. J. Angew. Chem. Int. Ed. 2011, 50, 10522.

(3) Golder, M. R.; Jasti, R. Acc. Chem. Res. 2015, 48, 557.

(4) Iyoda, M.; Shimizu, H. Chem. Soc. Rev. 2015, 44, 6411.

(5) Luan, Y.; Cong, H. Synlett 2017, 28, 1383.

(6) Miki, K.; Ohe, K. Chem. Eur. J. 2020, 26, 2529.

(7) Hermann, M.; Wassy, D.; Esser, B. Angew. Chem. Int. Ed. Engl. 2020. Doi: 10.1002 /anie.202007024.

(8) Chen, H.; Miao, Q. J. Phys. Org. Chem. 2020, 33, e4145.

(9) Tian, X.; Jasti, R. Cycloparaphenylenes: The Shortest Possible Segments of Armchair Carbon Nanotubes. InFragments of Fullerenes and Carbon Nanotubes: Designed Synthesis, Unusual
Reactions, and Coordination Chemistry. Petrukhina, M. A.; Scott, L. T. John Wiley \& Sons: Hoboken, 2011, 291-309.

(10) Leonhardt, E. J.; Jasti, R. Nat. Rev. Chem. 2019, 3, 672.

(11) Lewis, S. E. Chem. Soc. Rev. 2015, 44, 2221.

(12) Segawa, Y.; Levine, D. R.; Itami, K. Acc. Chem. Res. 2019, 52, 2760.

(13) Yamago, S.; Kayahara, E. J. Synth. Org. Chem., Jpn. 2019, 77, 1147.

(14) Qiu, Z.; Tang, C.; Wang, C.; Ju, Y.; Chun, K.; Deng, Z.; Hou, H.; Liu, Y.; Tan, Y. Angew. Chem. Int. Ed. 2020, 5, 9.

(15) Lovell, T.; Garrison, Z.; Jasti, R. Angew. Chem. Int. Ed. 2020, 59, 14363.

(16) Chen, M.; Yan, L.; Zhao, Y.; Murtaza, Y.; Meng, H.; Huang, W. J. Mater. Chem. C 2018, 6, 7416.

(17) Becker, H.-D. Chem. Rev. 1993, 93, 145.

(18) Danel, K.; Huang, T.-H.; Lin, J. T.; Tao, Y.-T.; Chuen, C.-H. Chem. Mater. 2002, 14, 3860.

(19) Islangulov, R. R.; Castellano, F. N. Angew. Chem. Int. Ed. 2006, 45, 5957.

(20) Zhou, J.; Liu, Q.; Feng, W.; Sun, Y.; Li, F. Chem. Rev. 2015, 115, 395.

(21) Ieuji, R.; Goushi, K.; Adachi, C. Nat. Commun. 2019, 10, 5283.

(22) Bharmoria, P.; Bildirir, H.; Moth-Poulsen, K. Chem. Soc. Rev. 2020, $49,6529$.

(23) Toyota, S.; Goichi, M.; Kotani, M. Angew. Chem. Int. Ed. 2004, 43, 2248.

(24) Chan, J. M. W.; Tischler, J. R.; Kooi, S. E.; Bulović, V.; Swager, T. M. J. Am. Chem. Soc. 2009, 131, 5659.

(25) Toyota, S. Chem. Lett. 2011, 40, 12.

(26) Yamamoto, Y.; Wakamatsu, K.; Iwanaga, T.; Sato, H.; Toyota, S. Chem. Asian J. 2016, 11, 1370.

(27) Yamamoto, Y.; Tsurumaki, E.; Wakamatsu, K.; Toyota, S. Angew. Chem. Int. Ed. 2018, 57, 8199.

(28) Toyota, S.; Yamamoto, Y.; Wakamatsu, K.; Tsurumaki, E.; MuñozCastro, A. Bull. Chem. Soc. Jpn. 2019, 92, 1721.

(29) Li, Z.; Sei, Y.; Akita, M.; Yoshizawa, M. Chem. Asian J. 2014, 9, 1016.

(30) Yazaki, K.; Sei, Y.; Akita, M.; Yoshizawa, M. Nat. Commun. 2014, $5,5179$.

(31) Giovannantonio, M. D.; Yao, X.; Eimre, K.; Urgel, J. I.; Ruffieux, P.; Pignedoli, C. A.; Müllen, K.; Fasel, R.; Narita, A. J. Am. Chem. Soc. 2020, 142, 12046.

(32) Takaki, Y.; Ozawa, R.; Kajitani, T.; Fukushima, T.; Mitsui, M.; Kobayashi, K. Chem. Eur. J. 2016, 22, 16760.

(33) Wang, J.; Zhuang, G.; Chen, M.; Lu, D.; Li, Z.; Huang, Q.; Jia, H.; Cui, S.; Shao, X.; Yang, S.; Du, P. Angew. Chem. Int. Ed. 2020, 59, 1619.

(34) Miyamoto, K.; Iwanaga, T.; Toyota, S. Chem. Lett. 2010, 39, 288.

(35) Matsuki, H.; Okubo, K.; Takaki, Y.; Niihori, Y.; Mitsui, M.; Kayahara, E.; Yamago, S.; Kobayashi, K. Angew. Chem. Int. Ed. Engl. 2020. Doi: 10.1002 /anie.202012120.

(36) Miki, K.; Fujita, M.; Inoue, Y.; Senda, Y.; Kowada, T.; Ohe, K. J. Org. Chem. 2010, 75, 3537.

(37) Miki, K.; Saiki, K.; Umeyama, T.; Baek, J.; Noda, T.; Imahori, H.; Sato, Y.; Suenaga, K.; Ohe, K. Small 2018, 14, 1800720.

(38) Guo, L.; Yang, X.; Cong, H. Chin. J. Chem. 2018, 36, 1135.

(39) Toyota, S.; Kurokawa, M.; Araki, M.; Nakamura, K.; Iwanaga, T. Org. Lett. 2007, 9, 3655.

(40) Toyota, S.; Onishi, H.; Wakamatsu, K.; Iwanaga, T. Chem. Lett. 2009, 38, 350.

(41) Toyota, S.; Oki, T.; Inoue, M.; Wakamatsu, K.; Iwanaga, T. Chem. Lett. 2015, 44, 978.

(42) Shirai, A.; Sano, H.; Nakamura, Y.; Takashika, M.; Otani, H.; Hasegawa, M.; Kato, S. I.; Iyoda, M. J. Org. Chem. 2018, 83, 3857. 
(43) Toyota, S.; Yoshikawa, M.; Saibara, T.; Yokoyama, Y.; Komori, T.; Iwanaga, T. ChemPlusChem 2019, 84, 643.

(44) Synthetic procedure for compound 1: A suspension of compound 5 (75 mg, $0.123 \mathrm{mmol}$ ), compound 4 (67.8 $\mathrm{mg}, 1.2$ equiv, $0.147 \mathrm{mmol}$ ), tetrakis(triphenylphosphine)palladium ( $28 \mathrm{mg}, 0.2$ equiv, $0.025 \mathrm{mmol}$ ), and potassium carbonate ( $68 \mathrm{mg}$, 4.0 equiv, $0.492 \mathrm{mmol})$ in potassium carbonate $(12 \mathrm{~mL})$ and $\mathrm{H}_{2} \mathrm{O}(2 \mathrm{~mL})$ was degassed by three freeze-pump-thaw cycles. The reaction mixture was heated at $80{ }^{\circ} \mathrm{C}$ overnight under nitrogen. After cooling to room temperature, the mixture was extracted with dichloromethane and washed with water. The organic layer was dried over anhydrous magnesium sulfate. The solvent was removed under vacuum and the residue was purified by silica gel column chromatography using hexane/dichloromethane $(30 / 1, v / v)$ as eluent to give the macrocycle compound 29,32dimesityl-10,13:23,26-dietheno-4,6:17,19-di(metheno)tetrabenzo[a,d,j,m][18]annulene $(\mathbf{1}, 59.2 \mathrm{mg}, 65 \%)$ as a white solid. ${ }^{1} \mathrm{H}$ NMR (400 MHz, methylene chloride-d2): $\delta 8.75$ (s, $2 \mathrm{H}$ ), 7.74 $(\mathrm{s}, 8 \mathrm{H}), 7.63-7.54(\mathrm{~m}, 8 \mathrm{H}), 7.52-7.49(\mathrm{~m}, 4 \mathrm{H}), 7.21(\mathrm{~s}, 4 \mathrm{H}), 2.53(\mathrm{~s}$, $6 \mathrm{H}), 1.85(\mathrm{~s}, 12 \mathrm{H}) .{ }^{13} \mathrm{CNMR}(101 \mathrm{MHz}$, methylene chloride-d2): $\delta$ 140.8, 139.7, 137.5, 137.3, 136.0, 134.8, 130.4, 130.2, 129.9, 128.3, 125.5, 125.3, 124.1, 20.9, 19.8. HRMS (EI): $m / z[M]+$ Calcd for $\mathrm{C}_{58} \mathrm{H}_{44}$ 740.3443; Found 740.3481. mp: $>350{ }^{\circ} \mathrm{C}$. IR: 3368.0, 2920.5, 2851.6, 1617.2, 1445.0, 1263.1, 1022.1, 820.5, 751.6, $682.7 \mathrm{~cm}^{-1}$.
(45) Synthetic procedure for compound 2: A suspension of compound 6 (75 mg, $0.123 \mathrm{mmol})$, compound 4 ( $67.8 \mathrm{mg}$, 1.2 equiv, $0.147 \mathrm{mmol}$ ), tetrakis(triphenylphosphine)palladium (28 $\mathrm{mg}$, 0.2 equiv, $0.025 \mathrm{mmol}$ ), and potassium carbonate $(68 \mathrm{mg}, 4.0$ equiv, $0.492 \mathrm{mmol}$ ) in potassium carbonate $(12 \mathrm{~mL})$ and $\mathrm{H}_{2} \mathrm{O}$ $(2 \mathrm{~mL})$ was degassed by three freeze-pump-thaw cycles. The reaction mixture was heated at $80{ }^{\circ} \mathrm{C}$ overnight under nitrogen. After cooling to room temperature, the mixture was extracted with dichloromethane and washed with water. The organic layer was dried over anhydrous magnesium sulfate. The solvent was removed under vacuum and the residue was purified by silica gel column chromatography using hexane/dichloromethane (30/1, $\mathrm{v} / \mathrm{v}$ ) as eluent to give the macrocycle compound 30,32-dimesityl4,6:10,14:18,20:24,28-tetra(metheno)tetrabenzo[ $a, d, k, n][20]$ annulene $(2,57 \mathrm{mg}, 63 \%)$ as a white solid. ${ }^{1} \mathrm{H}$ NMR $(400 \mathrm{MHz}$, methylene chloride-d2): $\delta 8.26(\mathrm{~s}, 2 \mathrm{H}), 7.61-7.57(\mathrm{~m}, 2 \mathrm{H})$, 7.55-7.44 (m, 10 H), 7.43-7.32 (m, 8 H), 7.18 (s, 2 H), 7.14 (s, $2 \mathrm{H})$, $2.49(\mathrm{~s}, 6 \mathrm{H}), 1.82(\mathrm{~s}, 6 \mathrm{H}), 1.71(\mathrm{~s}, 6 \mathrm{H}) .{ }^{13} \mathrm{C}$ NMR $(101 \mathrm{MHz}$, methylene chloride-d2): $\delta 141.1,140.9,137.4,137.3,135.8,134.8$, 131.1, 130.9, 129.5, 128.6, 128.4, 128.3, 128.2, 125.9, 125.4, 125.1, 124.2, 20.9, 19.7, 19.6. HRMS (ESI): $m / z$ [M] ${ }^{+}$Calcd for $\mathrm{C}_{58} \mathrm{H}_{45}$ 740.3443; Found 740.3498. $\mathrm{mp}:>350{ }^{\circ} \mathrm{C}$. IR: $2964.8,2925.4$, 2851.6, 1445, 1086, 889.4, 825.4, 751.6, 702.5, $623.7 \mathrm{~cm}^{-1}$.

(46) Goldberg,J. M.; Guard, L. M.; Wong, G. W.; Brayton, D. F.; Kaminsky, W.; Goldberg, K. I.; Heinekey, D. M. Organometallics 2020, 39, 3323. 